\title{
Ultrawideband Technology in Medicine: A Survey
}

\author{
R. Chávez-Santiago, ${ }^{1,2,3}$ I. Balasingham, ${ }^{1,2,3}$ and J. Bergsland ${ }^{1,2}$ \\ ${ }^{1}$ The Intervention Center, Oslo University Hospital, Sognsvannsveien 20, 0027 Oslo, Norway \\ ${ }^{2}$ Institute of Clinical Medicine, University of Oslo, 0316 Oslo, Norway \\ ${ }^{3}$ Department of Electronics and Telecommunications, Norwegian University of Science and Technology (NTNU), \\ 7491 Trondheim, Norway
}

Correspondence should be addressed to R. Chávez-Santiago, raul.chavez-santiago@rr-research.no

Received 30 November 2011; Accepted 1 February 2012

Academic Editor: Hsien-Chin Chiu

Copyright ( 2012 R. Chávez-Santiago et al. This is an open access article distributed under the Creative Commons Attribution License, which permits unrestricted use, distribution, and reproduction in any medium, provided the original work is properly cited.

\begin{abstract}
The utilization of wireless technology in traditional medical services provides patients with enhanced mobility. This has a positive effect on the recovery speed of a person after major surgical procedures or prolonged illness. Ultrawideband (UWB) radio signals have inherent characteristics that make them highly suitable for less invasive medical applications. This paper surveys our own and related recent research on UWB technology for medical sensing and communications. Some research perspectives in the aforementioned topics are suggested too.
\end{abstract}

\section{Introduction}

In recent years there has been an increasing interest in using ultra wideband (UWB) technology for short-range wireless communication interfaces [1]. The IEEE 802.15.4a standard has adopted UWB as one of the interfaces for robust low-data-rate transmission in wireless personal networks (WPANs) with precision ranging capabilities [2]. UWB signals have an inherent noise-like behavior due to their extremely low maximum effective isotropically radiated power (EIRP) spectral density of $-41.3 \mathrm{dBm} / \mathrm{MHz}$. This makes them difficult to detect and robust against jamming, potentially rescinding the need for complex encryption algorithms in tiny transceivers. Owing to these characteristics, UWB has emerged as a solution for the radio communication interface in medical wireless body area networks (WBANs) [3]. Additionally, UWB signals do not cause significant interference to other systems operating in the vicinity and do not represent a threat to patients' safety [4]. Impulse radio (IR) transceivers have simple structure and very low power consumption, which facilitates their miniaturization [5-7].

Another promising application of UWB as wireless communication interface is in capsule endoscopy. A capsule endoscope is a camera with the size and shape of a pill that is swallowed in order to visualize the gastrointestinal tract. They originally were devised to transmit still images of the digestive tract for subsequent diagnosis and detection of gastrointestinal diseases. Nevertheless, real-time video imaging of the digestive tract is feasible using an UWB radio interface $[8,9]$. Although capsule endoscopy is an invasive technique, its use is significantly more comfortable to the patient than traditional endoscopy and colonoscopy. Capsule endoscopes have demonstrated the ability to detect diseases in the small intestine in cases in which other techniques cannot [10-12].

Besides being used as wireless communication interface, UWB technology has many other possible applications in healthcare systems [13]. For instance, the IR-UWB radar has the potential to detect, noninvasively, tiny movements inside the human body [14]. Thereby, it is entirely possible to monitor cardiovascular physiological parameters using movement detection of the aorta [15-18] or other parts of the arterial system. Such parameters include heart rate (HR), respiration motion, blood pressure (BP), and so forth. Due to the architecture of the UWB radar, it is feasible to use the same electronics for both radar sensing and IR-UWB communications [7].

Imaging of surface and more deeply located structures such as breast tissue for cancer diagnosis is another promising application of UWB technology [19-25]. This has the potential of taking over the role of X-ray mammography. The 
great advantage of UWB is the absence of harmful effects due to long exposure to ionizing radiation. However, this application is not reviewed in here.

The rest of the paper is organized as follows. Section 2 describes the use of UWB as the communication interface for medical sensors in a WBAN. Section 3 is devoted to the use of UWB for capsule endoscopy. Section 4 surveys the research on UWB radar for medical sensing, particularly BP sensing. Finally, our conclusions are summarized in Section 5.

\section{Wireless Body Area Networks}

The integration of on-body medical sensors, in-body sensors, and UWB radars into a single network requires a carefully planned architecture in order to guarantee proper operation without mutual interference between the different devices. The Federal Communication Commission (FCC) has allocated the $3.1-10.6 \mathrm{GHz}$ frequency band for UWB communications in the United States [26]. It is important to notice, however, that a large part of this spectrum is strictly regulated in Europe and might not be available for new UWB radio systems. According to the European regulations $[27,28]$, only the $6-8.5 \mathrm{GHz}$ part of the spectrum is readily available for UWB transmissions without the use of interference mitigation techniques. Transmissions in 3.4-4.2 GHz and $8.5-9 \mathrm{GHz}$ are allowed for devices implementing the detect-and-avoid (DAA) interference mitigation technique. Beyond December 31, 2010, the $4.2-4.8 \mathrm{GHz}$ portion of the spectrum can be used if both DAA and low-duty-cycle (LDC) transmissions are implemented. Below is a description of a viable communication configuration of WBAN using different portions of the UWB spectrum.

2.1. Communication Interface for Implanted Sensors. Our research on UWB radio propagation inside the human body [29] revealed higher signal attenuation at higher frequencies. Since miniaturization and power consumption are the most important design constraints for in-body sensors, the signal attenuation through living tissues must be the minimal possible. Therefore, the $3.4-4.8 \mathrm{GHz}$ frequency band is the most appropriate for these devices [30]. Although the regulations request the implementation of DAA and LDC in this portion of the spectrum, it is very unlikely that radio signals radiated from inside the human body at extremely low power can cause interference to other NB systems. In the case of full-duplex links, DAA and LDC can be implemented in the on-body transceiver where more complex circuitry can be afforded. We have developed UWB propagation models for the communication of implanted sensors. One of these models provides the mathematical expression for the attenuation of a UWB channel between 1 and $6 \mathrm{GHz}$ in the chest [31], as shown below

$$
L_{[\mathrm{dB}]}(d)=10_{[\mathrm{dB}]}+0.987(d)^{0.85}+\mathcal{N}(0, \sigma),
$$

where $d$ is the depth from the skin in millimeters $(1<d<$ 120 ) and $\mathcal{N}$ is a Gaussian-distributed random variable with zero mean $\mu=0$ and standard deviation $\sigma=7.84$ that models the scattering around $L_{[\mathrm{dB}]}(d)$. In the same vein, an expression for the attenuation in the abdomen is given in [32] as

$$
L_{[\mathrm{dB}]}(d)=3.55_{[\mathrm{dB}]}+2.46(d)+\mathcal{N}(\mu(d), \sigma(d)),
$$

where $1<d<150 \mathrm{~mm}$. The values of $\mu(d)$ and $\sigma(d)$ are found in [32] for different depths.

The mathematical expressions of the statistical characterization for the channel impulse response (CIR) in both cases are provided in $[31,32]$, respectively. These models facilitate the reproduction of distorted UWB pulses after propagating through human tissues, which is essential for the design and evaluation of implanted transceivers.

\subsection{Communication Interface for On-Body Sensors. On-} body medical sensors (motes) operate on or in very close proximity to the patient's skin (typically less than 2 centimeters). Typical data rate requirements for common onbody medical sensing devices are given in [3]; electromyography (EMG) sensing is the most throughput-demanding application (up to $1500 \mathrm{kbps}$ ). However, it is important to note that continuous EMG monitoring is not necessary as is the case of electrocardiogram (ECG) and electroencephalogram (EEG) that require 10-100 kbps and 10-200 kbps, respectively. The IEEE 802.15.6 task group (http://www .ieee802.org/15/pub/TG6.html) has produced the first standard draft that details the characteristics of the UWB wireless interface for on-body sensors. IR-UWB on-body transceivers in the lower part of the UWB spectrum, that is, $3.4-4.8 \mathrm{GHz}$, are expected to be used. Experimental prototypes of these devices have already been developed $[28,33,34]$. On-body channels are more prone to experience fading and larger RMS delay spread than in-body channels [35]; thus, IR-UWB on-body transceivers are expected to support low data rate only [36].

Because of the complex geometry of the human body, it is likely that some on-body nodes require the relaying of their data when a single-hop link cannot be established because of extremely high signal attenuation. A relay node (RN) fulfils this task [28], although it is possible to include relaying capabilities in each mote's transceiver to ensure the establishment of multihop links whenever is required. Moreover, an implanted sensor needs a RN to transmit their data to a network controller for processing and displaying. This special RN is referred to as gateway node (GN).

2.3. Network Controller and Patient Monitor. The information collected by the low data rate sensors is gathered by a device known as body area network controller (BNC). The on-body sensors are connected to the BNC in a simple star network topology [37] using the IR-UWB 3.4-4.8 GHz onbody interface (Figure 1). The BNC can be devised as a personal digital assistant (PDA) that executes a MAC protocol, for example, [38], in order to ensure that all the sensors transmit their information in an organized and fair way.

For in-home healthcare, the BNC can display several basic vital signals such as temperature, heart rate (HR), blood 


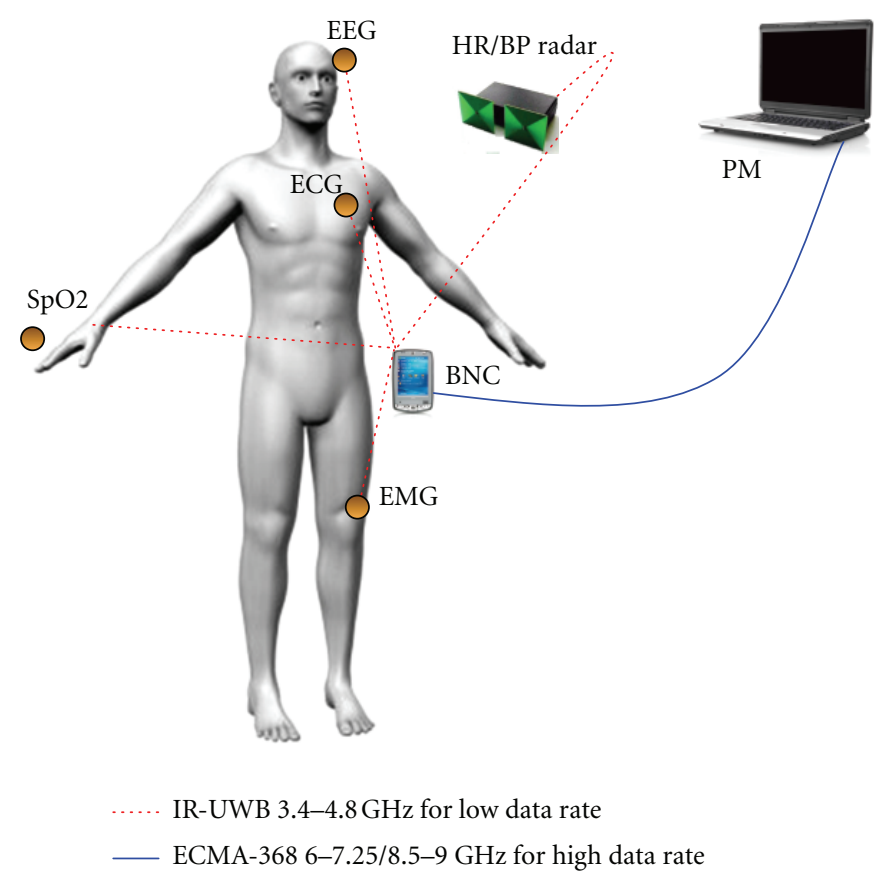

FIGURE 1: Network Topology of an UWB-WBAN.

pressure (BP), and oxygen saturation $\left(\mathrm{SpO}_{2}\right)$. However, for in-hospital healthcare and during surgery in an operating room (OR), a more powerful computer is necessary to display more complex signals such as ECG. This computer is referred to as patient monitor (PM) and is connected to the BNC through a high-data-rate UWB interface, namely, the ECMA-368 radio interface [39]. This interface, based on multiband orthogonal frequency division multiplexing (MBOFDM), can support $480 \mathrm{Mbps}$ within distances of up to $3 \mathrm{~m}$ and $110 \mathrm{Mbps}$ up to $10 \mathrm{~m}$. The link between the BNC and the PM can operate in the $6-7.25 \mathrm{GHz}$ and $8.5-9 \mathrm{GHz}$, which roughly translates into subbands 7,8 , and 11 , of the 14 subbands in which the UWB spectrum is divided according to the ECMA-368 standard. Other high-data-rate devices such as imaging medical radars can be connected to the PM using the same interface.

As seen, the BNC must support both IR-UWB in 3.4$4.8 \mathrm{GHz}$ and ECMA-368 in $6-7.25 \mathrm{GHz}$ and $8.5-9 \mathrm{GHz}$. Since DAA is required in $8.5-9 \mathrm{GHz}$, this part of the spectrum must be occupied mainly for temporary transmissions. The inherent capability for frequency agility of the ECMA-368 interface can be exploited to ensure the electromagnetic compatibility (EMC) of the WBAN with other electronic devices in the OR. The core of an OFDM transceiver is an inverse/direct fast Fourier transform (IFFT/FFT) engine. It has been demonstrated that the FFT engine can be effectively used as a spectrum analyzer with a frequency resolution of $4.125 \mathrm{MHz}$ thereby facilitating the implementation of DAA algorithms $[40,41]$. The architecture of a $\mathrm{BNC}$ with cognitive radio [42] capabilities for the BNC of a medical WBAN has been presented in [43].

\section{Capsule Endoscopy}

Traditional medical practice for the diagnosis of patients with disorders such as anal bleeding, Crohn's disease, Celiac disease, and intestinal tumors relies on the insertion of flexible tubes containing cameras to examine hard-to-reach parts of the digestive tract. This technique, however, can examine the upper portion of the digestive tract only, while colonoscopes help to visualize the lower part (colon). There is a large portion (approx. $6 \mathrm{~m}$ ) of the small intestine that cannot be inspected with these techniques. Capsule endoscopes help to fill this gap with significantly less discomfort for the patient.

State-of-the-art capsule endoscopes are swallowed with water, after which the patient puts a recorder belt on the waist. Some hours later (typically eight), medical staff look for abnormalities by reviewing a video created from the still images transmitted wirelessly from the capsule endoscope to the recorder belt. Adding the capability to transmit and analyze high-definition (HD) video in real time can provide further advantages to the medical staff for an accurate diagnosis. This additional capability, however, might increase the complexity of the circuitry and hence the power consumption of the capsule endoscope. The power consumption of a capsule endoscope must be as low as possible (in the order of $1 \mathrm{~mW}$ ) with a mandatory small physical size (around 300 cubic millimeters). Transmitting real-time video requires a high-transmission-rate communication link, for example, 73.8 Mbps for uncompressed VGA data [37]. All these requirements are difficult to meet using narrowband (NB) systems that operate in the medical implant communication 


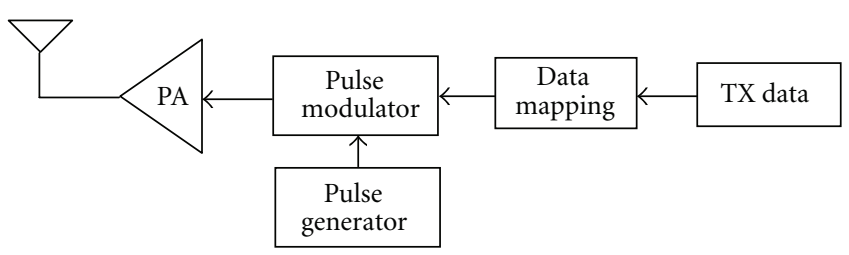

(a)

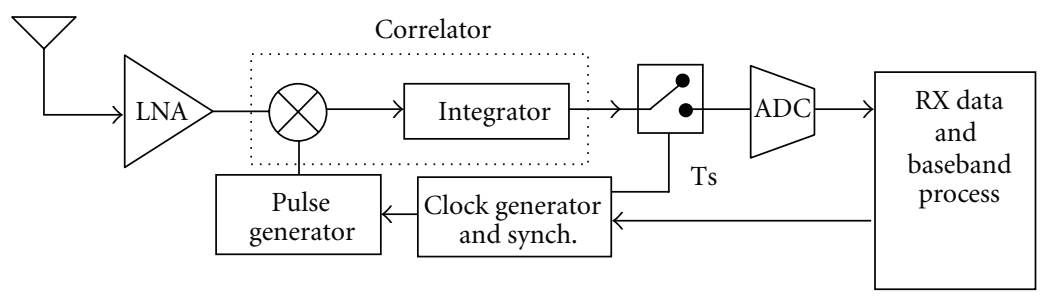

(b)

FIGURE 2: (a) In-body transmitter and (b) on-body receiver block diagrams for a capsule endoscope UWB communication system.

systems (MICS) frequency band of 401-406 MHz. In contrast, UWB technology has the potential to fulfill them all. In [9] we proposed the IR-UWB communication system for a capsule endoscope with high-data-rate capabilities. The UWB channel was characterized for the frequency range of 1-5 GHz using computer simulations. Nevertheless, we have recently developed a statistical UWB propagation model for capsule endoscope communications in 1-6 GHz [44].

3.1. In-Body Communication System Architecture. Due to the limitations at the in-body transmitter that include power consumption, size, system cost, and complexity, its communication architecture must be as simple as possible (Figure 2(a)). A pulse generator provides the UWB pulse that is subsequently modulated, amplified, and transmitted. The shape of the transmitted pulse determines the signal bandwidth. We used the fifth derivative of a Gaussian pulse to cover a bandwidth of approximately $1-5 \mathrm{GHz}$. The power spectral density (PSD) of the transmitted pulse is shown in Figure 3.

The generated data from the electrooptical circuitry of the capsule endoscope is directly modulated without further processing thereby simplifying the transmitter architecture. We considered the biphase pulse amplitude modulation (BPAM) scheme, in which the data bits are expressed by the polarity of the transmitted pulses. The resulting signal is then amplified and transmitted. The transmitter antenna must cover the entire frequency range with little pulse distortion. The design of a compact UWB antenna for the inbody transmitter is a challenging task, but some designs are available in the literature $[45,46]$.

3.2. On-Body Communication System Architecture. For the on-body receiver we proposed a novel architecture, which uses a single branch correlator (including a multiplier and an integrator) for recovering the transmitted signal. The block diagram of the receiver is depicted in Figure 2(b).

The UWB antenna at the receiver can be placed on the skin or at some distance away. By placing the receiving

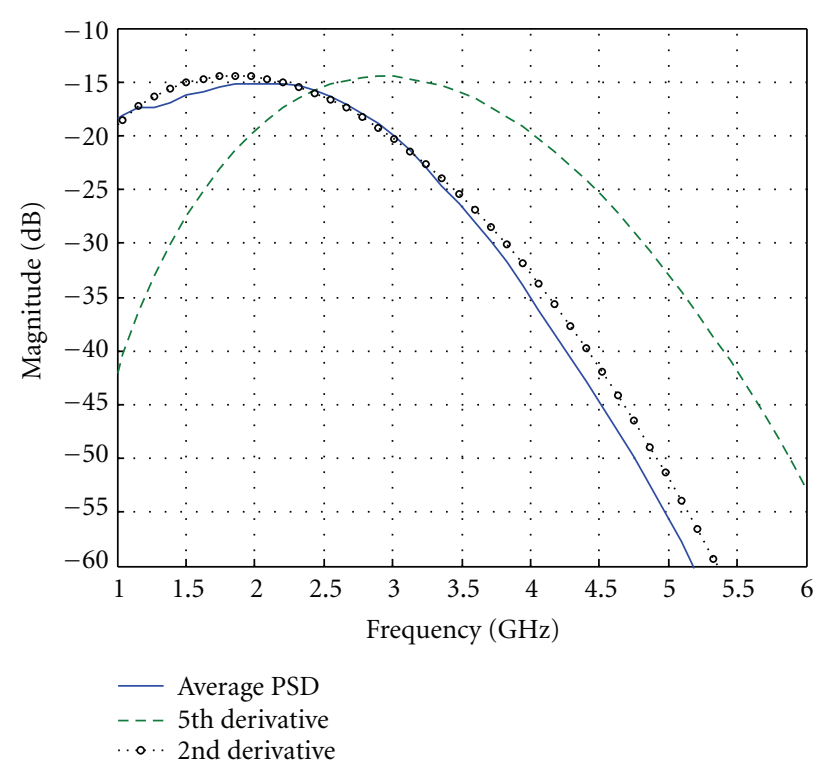

Figure 3: PSD of the transmitted Gaussian pulse (fifth derivative), the averaged PSD of the in-body channel, and the second derivative Gaussian pulse signal fitted to the average PSD.

antenna on the body surface, the nonradiative near-field components can be collected by the antenna thus improving the link quality significantly. We have found an improvement of $6 \mathrm{~dB}$ due to near-field coupling in UWB in-body links [47]. The practical implementation of the receiver antenna requires a special structure since it must cover a relatively wide body area (abdominal torso) [48]. Commonly, a spatial-diversity antenna array around the torso is embedded in a recorder belt, which is worn by the patient while the capsule endoscope operates.

The low-noise amplifier (LNA) increases the power of the received pulses to a suitable level for signal processing and to overcome noise in subsequent electronic stages. The data are subsequently recovered by the correlator. The correlation operation can be implemented in either analog 


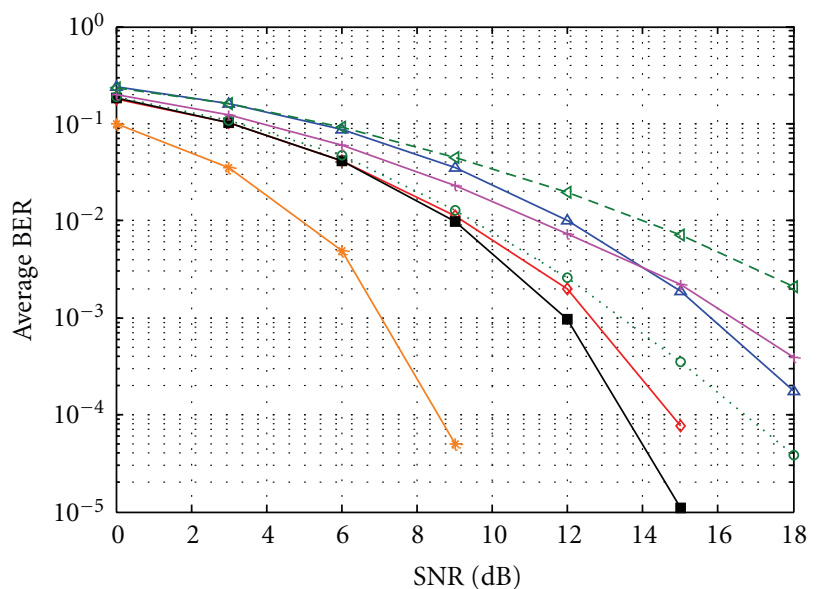

$$
\begin{array}{ll}
\triangle \text { Gaussian } & +4 \text { th derivative } \\
\neg-\text { 1st derivative } & -\varangle-5 \text { th derivative } \\
\rightarrow-\text { 2nd derivative } & \rightarrow \text { BPAM } \\
\therefore \odot \text { 3rd derivative } &
\end{array}
$$

FIGURE 4: Averaged BER performance using different templates.

or digital circuits. Using an all-digital receiver requires highly sophisticated receiver processors with an analog-to-digital converter (ADC) with sampling rate of 2 or 4 times that of the pulse bandwidth and resolution of 4-6 bits. A hybrid analog and digital receiver can reduce the system complexity and cost by decreasing the sampling rate and resolution of the ADC [49]. The correlator output is then sampled, and the ADC converts the analog-demodulated signal into digital form. The digital baseband circuitry provides control for the clock generation, synchronization, and data processing.

One might think that the receiver can take advantage of multipath signals by creating a bank of correlators (rake receiver structure). This idea has been applied to IR-UWB links in dispersive channels with large number of correlators and a more complicate system. However, the imperfect correlations resulting from distorted received pulses reduce the system performance. An optimal way to correct this problem is using a template-match detection technique that performs a matched filter operation with a series of template waveforms. However, the system complexity increases significantly, and channel estimation is required. Hence, we propose using a single branch correlator with an optimized predefined template that guarantees maximum energy recovery. The associated delay of the template is adjusted so that maximum correlator output at one branch is generated. The short rootmean-square (RMS) delay spread of in-body channels (in the order of $1 \mathrm{~ns}$ ) [29] enables this simple architecture.

The design of the pre-defined template depends on the propagation channel characteristics. By multiple electromagnetic (EM) simulations of UWB signals propagating through the abdomen, the normalized average PSD of the "digestive" radio channel was obtained (see Figure 3). The second derivative of a Gaussian pulse can approximate fairly well the PSD of the channel and therefore was chosen as the pre-defined template. It is important to mention, however, that this template pulse choice is optimal for the ideal case that we considered, that is, when the antenna effects are disregarded. Taking this into account, the antenna effects would have a considerable impact on the optimal template. In such case, the EM simulations must include the particular antenna specifications in order to select the most appropriate template for any other specific design.

3.3. Performance Evaluation. The average bit-error-rate (BER) performance (averaged over 90 arbitrary channel realizations) for different templates in an additive white Gaussian noise environment is compared in Figure 4. The worst performance is observed using the fifth derivative of the Gaussian pulse as template. The reduced BER performance reveals significant distortion of the transmitted pulse while propagating through the body tissues. The best BER performance is obtained for the second derivative, which collects more signal energy from the distorted pulses. For a typical BER of $10^{-3}$, a mismatch loss of $5 \mathrm{~dB}$ is observed with respect to the ideal case. Using the first and the third derivatives provides almost similar BER performance.

3.4. Ongoing Research. We recently carried out an experiment that demonstrated the feasibility of transmitting highdata-rate video $(\mathrm{H} .264 / 1280 \times 720$ pixels at 30 frames/s) through the living tissues of a porcine surgical model. The in-body to on-body communication was done using an ECMA-368 link in 4.2-4.8 GHz. Proper video transmission at $80 \mathrm{Mbps}$ was achieved at depths from the skin of up to $35 \mathrm{~mm}$. Further improvement is expected using lower frequencies and an implantable antenna [46]. Moreover, if the data from the electrooptical circuitry of the capsule endoscope is properly encoded [50], significant reduction of the required transmission rate can be obtained thereby improving the communication conditions. These speculations will be verified in future experiments.

One of our goals is the development of a full-duplex communication link for capsule endoscopy. This means integrating not only a transmitter but also a receiver (transceiver architecture) in the capsule endoscope. This will allow transmitting external commands (movement, optical focus on specific areas, etc.) to the capsule endoscope thereby adding more control and flexibility to the device. The same transceiver can be used to remotely control microrobotic multifunctional endoscopic devices, capable of performing several diagnostic and therapeutic operations such as biopsy, electrocautery, laser microsurgery, and so forth, with a retractable arm [51].

Accurate tracking of the capsule is required for all the aforementioned applications. However, the tracking problem is rather complicate due to the highly nonhomogeneous structure of the human body. Nevertheless, our research has demonstrated that the use of multimodel (MM) target tracking methods can provide accuracy in-body tracking in the millimeter scale $[52,53]$.

\section{Medical Radar}

Heart rate, respiration rate (RR), and heart movements recording have already been proved feasible using IR-UWB 


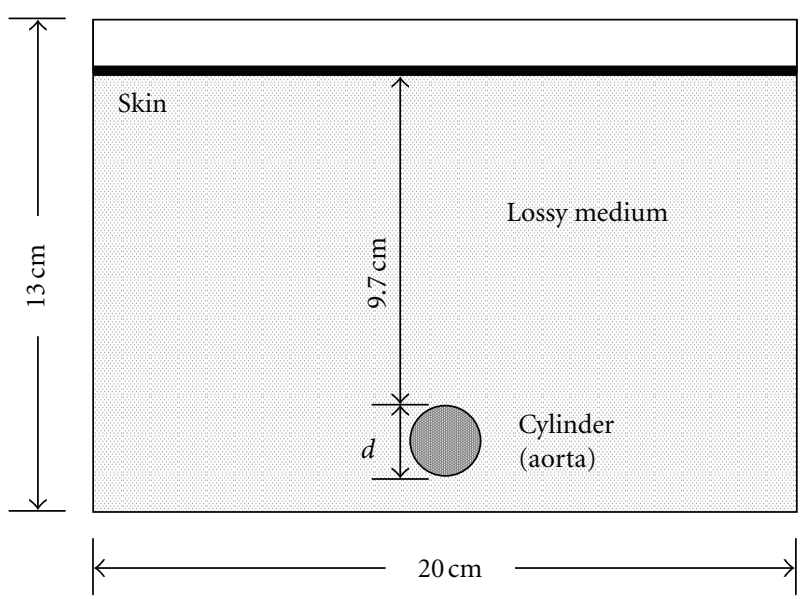

FIgURE 5: 2D simulation model for aorta's diameter estimation.

radar [54, 55]. Several other possible medical applications of UWB radar include ambulatory cardiac output monitoring, blood vessel movement recording, blood pressure celerity measurement, and shock diagnosis in emergency patients. Similar technology can obviously be applied to pneumology and polysomnography for apnoea monitoring in infants, obstructive sleep apnoea monitoring, allergy and asthma crisis monitoring, and so forth. The application of UWB radar in obstetrics as a replacement for ultrasound has also been proposed [14], but this idea has been looked upon cautiously because of the great concern regarding radiofrequency (RF) safety for the newborn. Nevertheless, UWB radar can offer the medical staff and patients several advantages over ultrasound, such as noncontact operation, no need for cleaning after use, remote and continuous operation, lower cost, and easier operation.

4.1. Blood Pressure Measurement. Noninvasive measurements of BP exist such as sphygmomanometer, photoplethysmograph [56], tonography [57], and pulse transit time [58]; however, they all rely on peripheral measurement points. This may constitute a problem in certain situations such as when flow redistribution to central parts of the body (heavy injury, temperature) degrades these measurements; another situation where central measurements may prove advantageous is in the presence of strong movement of the peripheral locations, which affects pressure measurements [59].

The use of radar techniques to measure BP may draw upon ideas from these fields, as well as from ground-penetrating radar (GPR), yet is different enough to merit a specific approach. In particular, the complexity of geometry and stronger attenuation are more significant in BP measurement compared with detection of breast cancer and HR and RR, which are essentially based on shallow reflections.

Estimating BP using radar techniques is necessarily indirect; pressure only affects propagation through the geometry and not material dielectric properties, contrarily to medical imaging using UWB radar for early breast cancer detection. The latter involves transmitting an extremely short pulse through the breast tissues and then recording the backscattered signal from different locations. The basis for detecting and locating a cancerous tumor is the different dielectric properties of healthy and malignant breast tissue. Healthy tissue is largely transparent to microwaves, whereas tumors, which contain more water and blood, scatter them back to the probing antenna array [22]. However, in the case of the aortic BP, two effects may relate aorta diameter (geometry) to its pressure:

(i) using the linear relationship between percentage changes in instantaneous BP and diameter, shown for carotid artery pressure in [60];

(ii) estimating the elasticity of the aorta (local compliance or incremental elastic modulus) and relating this to $\mathrm{BP}[58,59,61]$ without being explicit with respect to the functional relationship.

In both approaches, the radar-based method aims at detecting the aorta walls and estimates the diameter as a function of time.

From a medical point of view, central measurements are better than peripheral ones. Therefore, we pursued the measurement of BP through movement detection of the aorta. In order to understand the principles of using UWB radar to measure aorta diameter variations, a simple model was constructed for EM simulations [15]. Our model combines a voxel representation of the human body with the material dielectric properties proposed in [62]. It is based on a $2 \mathrm{D}$ simplified geometry: a cylinder of diameter $d$ (representing the aorta) immersed in a lossy medium (Figure 5). The lossy medium approximates average living tissue dielectric properties, except for the skin and aorta, the properties of which were taken from [62]. Further details of the model and the EM simulations can be found in [15].

With diameter variations in the order of $2 \mathrm{~mm}$, a set of simulations with aorta diameter ranging from $20 \mathrm{~mm}$ to $26 \mathrm{~mm}$ in steps of $0.4 \mathrm{~mm}$ were conducted in a simulation space with a resolution of $0.1 \mathrm{~mm}$. The current source signal in the simulations was the seventh derivative of a Gaussian pulse with energy centered around $4.5 \mathrm{GHz}$. This relatively high-order derivative was used for compensating, to a certain extent, the frequency-dependant attenuation in the simulations.

The analysis of the resulting transfer function and the time-domain echoes led to the conclusion that the backscattered signal from the aorta contains necessary information for distinguishing front and rear walls of the aorta thereby making the estimation of its diameter feasible. However, due to strong attenuation in living tissues, feasibility is essentially hinged on a viable power budget. In the simulations, an upper bound on received power in the $0.8-5 \mathrm{GHz}$ range shows a $40 \mathrm{~dB}$ loss at the lower end increasing to about $120 \mathrm{~dB}$ at the upper end where material loss is dominant.

There are several tradeoffs involved in achieving BP measurements with UWB radar: high-frequency content versus SNR at the receiver as tissues generally severely attenuate the signals; high-frequency versus resolution (and signal-to-clutter reduction) for the same reason. A criterion 
for the best selection of bandwidth and center frequency was presented in $[16,17]$. Even with a more realistic simulation scenario than that in Figure 5, the feasibility of estimating the aorta radius with the use of UWB radar has been demonstrated [18].

Another key issue that needs to be addressed is whether the use of an antenna array improve measurements, and if so, how much and in which way should this best be implemented. Potentially, the use of an array introduces spatial selectivity and may improve the signal-to-clutter ratio.

Our ongoing research toward the demonstration of BP measurements using UWB radar is focused on measurements with a phantom model that mimics a complex geometry for the estimation of the aorta diameter with sets of static measurements. This will allow for direct comparison between theoretic and practical results.

\section{Conclusions}

Ultra wideband technology has many potential applications in medicine for less invasive medical diagnosis and monitoring. The UWB radar can potentially be used in novel noninvasive sensing and imaging techniques owing to its high temporal resolution for detecting backscattered signals. We have described our current research on the application of this technology to noninvasive measurement of blood pressure.

The other application of UWB is as low-power wireless communication interface. Particularly, impulse radio seems to perfectly fit the communication requirements of tiny medical sensors, including in-body ones. Our research in this area was presented through the case study of a capsule endoscope system. Additionally, medical sensors and radars can be interconnected using UWB interfaces thereby enhancing the mobility of patients during surgery or intensive therapy. We described the integration architecture of all these systems into a single wireless body area network. One major issue to consider while interconnecting several medical devices using UWB radio interfaces is the possibility of mutual interference with other systems that already operate in the $3.1-10.6 \mathrm{GHz}$ frequency band. Therefore, new interference avoidance techniques and frequency agility such as cognitive radio have to be investigated.

These two aspects of UWB (radar and wireless communi-cation interface) for medical applications are being investigated in Norway by the MELODY Project (http://www .melody-project.info/); MELODY stands for "medical sensing, localization, and communications using ultra wideband technology." The ultimate objective of this project is the improvement of current wireless health systems and the possible development of novel medical applications based on UWB technology.

\section{Acknowledgment}

This work is part of the MELODY Project, which is funded by the Research Council of Norway under the Contract no. $187857 / S 10$.

\section{References}

[1] C. C. Chong, F. Watanabe, and H. Inamura, "Potential of UWB technology for the next generation wireless communications," in Proceedings of the 9th IEEE International Symposium on Spread Spectrum Techniques and Applications (ISSSTA '06), pp. 422-429, Manaus, Amazon, Brazil, August 2006.

[2] J. Zhang, P. V. Orlik, Z. Sahinoglu, A. F. Molisch, and P. Kinney, "UWB systems for wireless sensor networks," Proceedings of the IEEE, vol. 97, no. 2, pp. 313-331, 2009.

[3] P. Gandolfo, D. Radović, M. Savić, and D. Simić, "IEEE 802.15.4a UWB-IR radio system for telemedicine," in Proceeedings of the IEEE International Conference on Ultra-Wideband (ICUWB '08), vol. 3, pp. 11-14, Hannover, Germany, September 2008.

[4] M. Hämäläinen, P. Pirinen, J. Iinatti, and A. Taparugssanagorn, "UWB supporting medical ICT applications," in Proceeedings of The IEEE International Conference on Ultra-Wideband (ICUWB '08), vol. 3, pp. 15-16, Hannover, Germany, September 2008.

[5] A. P. Chandrakasan, F. S. Lee, D. D. Wentzloff et al., "Lowpower impulse UWB architectures and circuits," Proceedings of the IEEE, vol. 97, no. 2, pp. 332-352, 2009.

[6] J. Ryckaert, C. Desset, A. Fort et al., "Ultra-wide-band transmitter for low-power wireless body area networks: design and evaluation," IEEE Transactions on Circuits and Systems I, vol. 52, no. 12, pp. 2515-2525, 2005.

[7] C. Y. Lee and C. Toumazou, "Ultra-low power UWB for real time biomedical wireless sensing," in Proceedings of the IEEE International Symposium on Circuits and Systems (ISCAS '05), pp. 57-60, Kobe, Japan, May 2005.

[8] M. M.-O. Lee, E. -M. Lee, B. L. Cho, K. Eshraghian, and Y. -H. Kim, "The UTCOMS: a wireless video capsule nanoendoscope," in Endoscopic Microscopy, vol. 6082 of Proceedings of SPIE, pp. 60820F.1-60820F.10, San Jose, Calif, USA, January 2006.

[9] A. Khaleghi, R. Chávez-Santiago, and I. Balasingham, "Ultrawideband pulse-based data communications for medical implants," IET Communications, vol. 4, no. 15, pp. 1889-1897, 2010.

[10] J. M. Herrerías, A. Caunedo, M. Rodríguez-Téllez, F. Pellicer, and J. M. Herrerías, "Capsule endoscopy in patients with suspected Crohn's disease and negative endoscopy," Endoscopy, vol. 35, no. 7, pp. 564-568, 2003.

[11] M. Mylonaki, A. Fritscher-Ravens, and P. Swain, "Wireless capsule endoscopy: a comparison with push enteroscopy in patients with gastroscopy and colonoscopy negative gastrointestinal bleeding," Gut, vol. 52, no. 8, pp. 1122-1126, 2003.

[12] PHYsorg.com, "Capsule endoscopy turning up undiagnosed cases of Crohn's disease," October 2007, http://www.physorg .com/pdf111757757.pdf.

[13] Y. Xu, Y. Lu, H. Zhang, and Y. Wang, "An overview of ultrawideb and technique application for medial engineering," in Proceedings of the IEEE/ICME International Conference on Complex Medical Engineering (CME'07), pp. 408-411, Beijing, China, May 2007.

[14] E. M. Staderini, "UWB radars in medicine," IEEE Aerospace and Electronic Systems Magazine, vol. 17, no. 1, pp. 13-18, 2002.

[15] L. E. Solberg, I. Balasingham, S. E. Hamran, and E. Fosse, "A feasibility study on aortic pressure estimation using UWB radar," in Proceedings of the IEEE International Conference on Ultra-Wideband (ICUWB '09), pp. 464-468, Vancouver, Canada, September 2009. 
[16] L.-E. Solberg and I. Balasingham, "Candidate estimators for aorta diameter estimation using bistatic radar," in Proceedings of the 5th International Conference on Body Area Networks (BodyNets '10), Corfu, Greece, September 2010.

[17] L. E. Solberg, S. E. Hamran, T. Berger, and I. Balasingham, "Minimum variance signal selection for aorta radius estimation using radar," EURASIP Journal on Advances in Signal Processing, vol. 2010, Article ID 682037, 13 pages, 2010.

[18] L.-E. Solberg, S.-E. Hamran, and I. Balasingham, "Realistic simulations of aorta radius estimation," in Proceedings of the IEEE 4th Int Symposium on Applied Sciences in Biomedical and Communication Technologies (ISABEL '11), Barcelona, Spain, October 2011.

[19] W. Liu, H. M. Jafari, S. Hranilovic, and M. J. Deen, "Time domain analysis of UWB breast cancer detection," in Proceedings of the 23rd Biennial Symposium on Communications, pp. 336-339, Kingston, Canada, May-June 2006.

[20] Y. Chen, E. Gunawan, Y. Kim, K. S. Low, C. B. Soh, and L. L. Thi, "UWB microwave breast cancer detection: generalized models and performance prediction," in Proceedings of the 28th Annual International Conference of the IEEE Engineering in Medicine and Biology Society (EMBS '06), pp. 2630-2633, New York, NY, USA, August-September 2006.

[21] C. Yifan, E. Gunawan, K. Yongmin, L. Kaysoon, and S. Cheongboon, "UWB microwave imaging for breast cancer detection: tumor/clutter identification using a time of arrival data fusion method," in Proceedings of the IEEE Antennas and Propagation Society International Symposium (APS '06), pp. 255-258, Albuquerque, NM, USA, July 2006.

[22] W. C. Khor and M. E. Bialkowski, "Investigations into an UWB microwave radar system for breast cancer detection," in Proceedings of the IEEE Antennas and Propagation Society International Symposium (AP-S '07), pp. 2160-2163, Honolulu, Hawaii, USA, June 2007.

[23] X. Xiao and T. Kikkawa, "Early breast cancer detection by ultrawide band imaging with dispersion consideration," Japanese Journal of Applied Physics, vol. 47, no. 4, pp. 3209-3213, 2008.

[24] X. Xiao and T. Kikkawa, "Influence of the organism interface on the breast cancer detection by UWB," Applied Surface Science, vol. 255, no. 2, pp. 597-599, 2008.

[25] S. A. AlShehri and S. Khatun, "UWB imaging for breast cancer detection using neural network," Progress in Electromagnetic Research C, vol. 7, pp. 79-93, 2009.

[26] FCC, "First report and order, revision of part 15 of the commission's rules regarding ultra-wideband transmission systems," ET Docket 98-153, FCC, 2002.

[27] E. Faussurier, "Spectrum management and ultra-wideband (UWB)," September 2008, http://www.icuwb2008.org/files/ files/pdf/Article-IEUWB_EFA-12sept2008.pdf.

[28] H.-B. Li, "Body area network-standardization and technology," in Proceedings of the 2nd International Symposium on Applied Sciences in Biomedical and Communication Technologies (ISABEL '09), Bratislava, Slovak Republic, November 2009.

[29] A. Khaleghi, R. Chávez-Santiago, X. Liang, I. Balasingham, V. C. M. Leung, and T. A. Ramstad, "On ultra wideband channel modeling for in-body communications," in Proceedings of the IEEE 5th International Symposium on Wireless Pervasive Computing (ISWPC '10), pp. 140-145, Modena, Italy, May 2010.

[30] Q. Wang, K. Masami, and J. Wang, "Channel modeling and BER performance for wearable and implant UWB body area links on chest," in Proceedings of the IEEE International Conference on Ultra-Wideband (ICUWB '09), pp. 316-320, Vancouver, Canada, September 2009.

[31] A. Khaleghi, R. Chávez-Santiago, and I. Balasingham, "Ultrawideband statistical propagation channel model for implant sensors in the human chest," IET Microwaves, Antennas \& Propagation, vol. 5, no. 15, pp. 1805-1812, 2011.

[32] S. Støa, R. Chavez-Santiago, and I. Balasingham, "An ultra wideband communication channel model for the human abdominal region," in Proceedings of the IEEE Global Communication Conference (Globecom '10), pp. 246-250, Miami, Fla, USA, December 2010.

[33] O. Lauer, D. Barras, M. Zahner, and J. Fröhlich, "Investigations of an IR-UWB based hardware demonstrator for wireless patient monitoring," in Proceedings of the Asia-Pacific Symposium \& Exhibition on Electromagnetic Compatibility (APEMC '11), Jeju Island, South Korea, May 2011.

[34] J. Shi and J. Wang, "A feasibility study of in-body to on-body transmission with IR-UWB transceiver," in Proceedings of the Asia-Pacific Symposium \& Exhibition on Electromagnetic Compatibility (APEMC '11), Jeju Island, South Korea, May 2011.

[35] IEEE P802.15-08-0780-09-0006, "Channel Model for Body Area Network (BAN)," April 2009.

[36] I. Dotlić and R. Miura, "Low data-rate ultra-wideband impulse radio communications for wireless medical body area networks," in Proceedings of the 4th International Symposium on Applied Sciences in Biomedical and Communication Technologies (ISABEL '11), Barcelona, Spain, October 2011.

[37] R. Chávez-Santiago, A. Khaleghi, I. Balasingham, and T. A. Ramstad, "Architecture of an ultra wideband wireless body area network for medical applications," in Proceedings of the 2nd International Symposium on Applied Sciences in Biomedical and Communication Technologies (ISABEL '09), Bratislava, Slovak Republic, November 2009.

[38] L. Kynsijärvi, L. Goratti, R. Tesi, J. Iinatti, and M. Hämäläinen, "Design and performance of contention based MAC protocols in WBAN for medical ICT using IR-UWB," in Proceedings of the IEEE International Symposium on Personal, Indoor and Mobile Radio Communications (PIMRC '10), pp. 107-111, Istanbul, Turkey, September 2010.

[39] ECMA-368 Standard, "High Rate Ultra Wideband PHY and MAC Standard,” December 2008.

[40] A. Batra, S. Lingam, and J. Balakrishnan, "Multi-band OFDM: a cognitive radio for UWB," in Proceedings of the IEEE International Symposium on Circuits and Systems (ISCAS '06), pp. 4094-4097, Island of Kos, Greece, May 2006.

[41] J. Lansford, "The WiMedia UWB radio: is it the ideal cognitive radio processor?" in Proceeedings of the IEEE International Conference on Ultra-Wideband (ICUWB '08), vol. 2, pp. 173176, Hannover, Germany, September 2008.

[42] N. Devroye, M. Vu, and V. Tarokh, "Cognitive radio networks: highlights of information theoretic limits, models, and design," IEEE Signal Processing Magazine, vol. 25, no. 6, pp. 1223, 2008.

[43] R. Chávez-Santiago and I. Balasingham, "Cognitive radio for medical wireless body area networks," in Proceedings of the IEEE 16th International Workshop on Computer Aided Modeling and Design of Communication Links and Networks (CAMAD '11), pp. 148-152, Kyoto, Japan, June 2011.

[44] S. Støa, R. Chavez-Santiago, and I. Balasingham, "An ultra wideband communication channel model for capsule endoscopy," in Proceedings of the 3rd International Symposium on Applied Sciences in Biomedical and Communication Technologies (ISABEL '10), Rome, Italy, November 2010. 
[45] T. Dissanayake, M. R. Yuce, and C. Ho, "Design and evaluation of a compact antenna for implant-to-air UWB communication," IEEE Antennas and Wireless Propagation Letters, vol. 8, pp. 153-156, 2009.

[46] Q. Wang, K. Wolf, and D. Plettemeier, "An UWB capsule endoscope antenna design for biomedical communications," in Proceedings of the 3rd International Symposium on Applied Sciences in Biomedical and Communication Technologies (ISABEL '10), Rome, Italy, November 2010.

[47] A. Khaleghi and I. Balasingham, "Improving in-body ultra wideband communication using near-field coupling of the implanted antenna," Microwave and Optical Technology Letters, vol. 51, no. 3, pp. 585-589, 2009.

[48] Q. Wang, R. Hahnel, H. Zhang, and D. Plettemeier, "Wearable Vivaldi UWB planar antenna for in-body communication," in Proceedings of the 4th IEEE International Symposium on Applied Sciences in Biomedical and Communication Technologies (IEEE ISABEL '11), Barcelona, Spain, October 2011.

[49] R. Gharpurey and P. Kinget, Ultra Wideband: circuits, Transceivers and Systems, Springer, New York, NY, USA, 2008.

[50] A. N. Kim, T. A. Ramstad, and I. Balasingham, "Very low complexity low rate image coding for the wireless endoscope," in Proceedings of the 4th IEEE International Symposium on Applied Sciences in Biomedical and Communication Technologies (IEEE ISABEL '11), Barcelona, Spain, October 2011.

[51] M. O. Schurr, S. Schostek, C. N. Ho, F. Rieber, and A. Menciassi, "Microtechnologies in medicine: an overview," Minimally Invasive Therapy and Allied Technologies, vol. 16, no. 2, pp. 76-86, 2007.

[52] B. Moussakhani, R. Chávez-Santiago, and I. Balasingham, "Multi model tracking for localization in wireless capsule endoscope," in Proceedings of the 4th IEEE International Symposium on Applied Sciences in Biomedical and Communication Technologies (IEEE ISABEL '11), Barcelona, Spain, October 2011.

[53] B. Moussakhani, J. T. Flåm, S. Støa, I. Balasingham, and T. A. Ramstad, "On localization accuracy inside human abdomen region,” IET Wireless Sensor Systems, vol. 2, no. 1, pp. 9-15, March 2012.

[54] C. G. Bilich, "Bio-medical sensing using ultra wideband communications and radar technology: a feasibility study," in Proceedings of the 1st International Pervasive Health Conference and Workshops, PervasiveHealth, pp. 1-9, NovemberDecember 2006.

[55] A. Lazaro, D. Girbau, and R. Villarino, "Analysis of vital signs monitoring using an IR-UWB radar," Progress in Electromagnetics Research, vol. 100, pp. 265-284, 2010.

[56] G. Parati, G. Ongaro, G. Bilo et al., "Non-invasive beat-tobeat blood pressure monitoring: new developments," Blood Pressure Monitoring, vol. 8, no. 1, pp. 31-36, 2003.

[57] K. Matthys and P. Verdonck, "Development and modelling of arterial applanation tonometry: a review," Technology and Health Care, vol. 10, no. 1, pp. 65-76, 2002.

[58] J. Y. A. Foo and S. L. Chu, "Pulse transit time as an indirect marker for variations in cardiovascular related reactivity," Technology and Health Care, vol. 14, no. 2, pp. 97-108, 2006.

[59] G. Sharwood-Smith, J. Bruce, and G. Drummond, "Assessment of pulse transit time to indicate cardiovascular changes during obstetric spinal anaesthesia," British Journal of Anaesthesia, vol. 96, no. 1, pp. 100-105, 2006.

[60] M. Sugawara, K. Niki, H. Furuhata, S. Ohnishi, and S. Suzuki, "Relationship between the pressure and diameter of the carotid artery in humans," Heart and Vessels, vol. 15, no. 1, pp. 49-51, 2000.
[61] J. Lass, K. Meigas, D. Karai, R. Kattai, J. Kaik, and M. Rossmann, "Continuous blood pressure monitoring during exercise using pulse wave transit time measurement," in Proceedings of the 26th Annual International Conference of the IEEE Engineering in Medicine and Biology (IEMBS '04), vol. 1, pp. 2239-2242, San Francisco, Calif, USA, September 2004.

[62] C. Gabriel and S. Gabriel, "Compilation of the dielectric properties of body tissues at RF and microwave frequencies," Tech. Rep. WC2R 2LS, Department of Physics, King's College, London, UK, June 1996. 

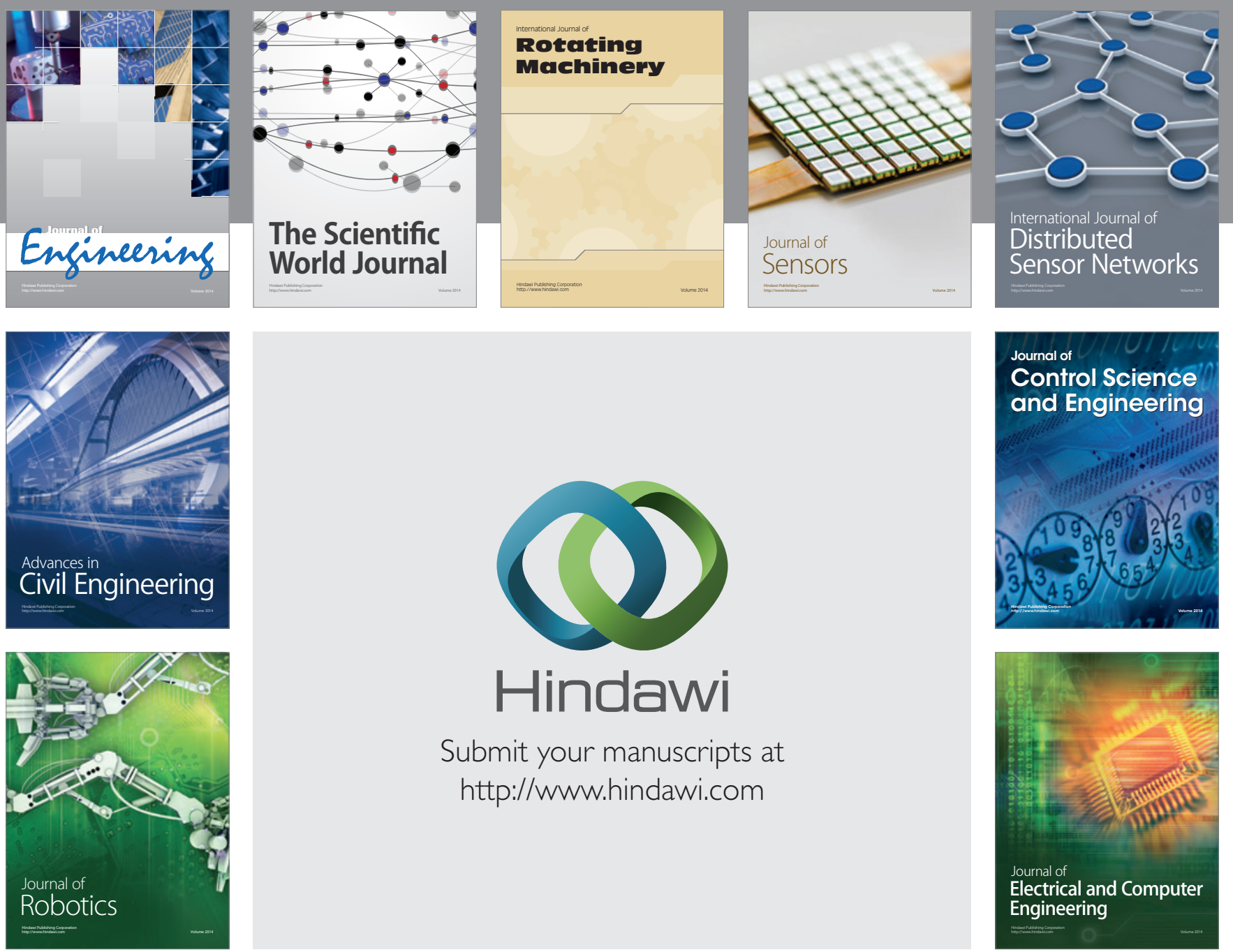

Submit your manuscripts at

http://www.hindawi.com
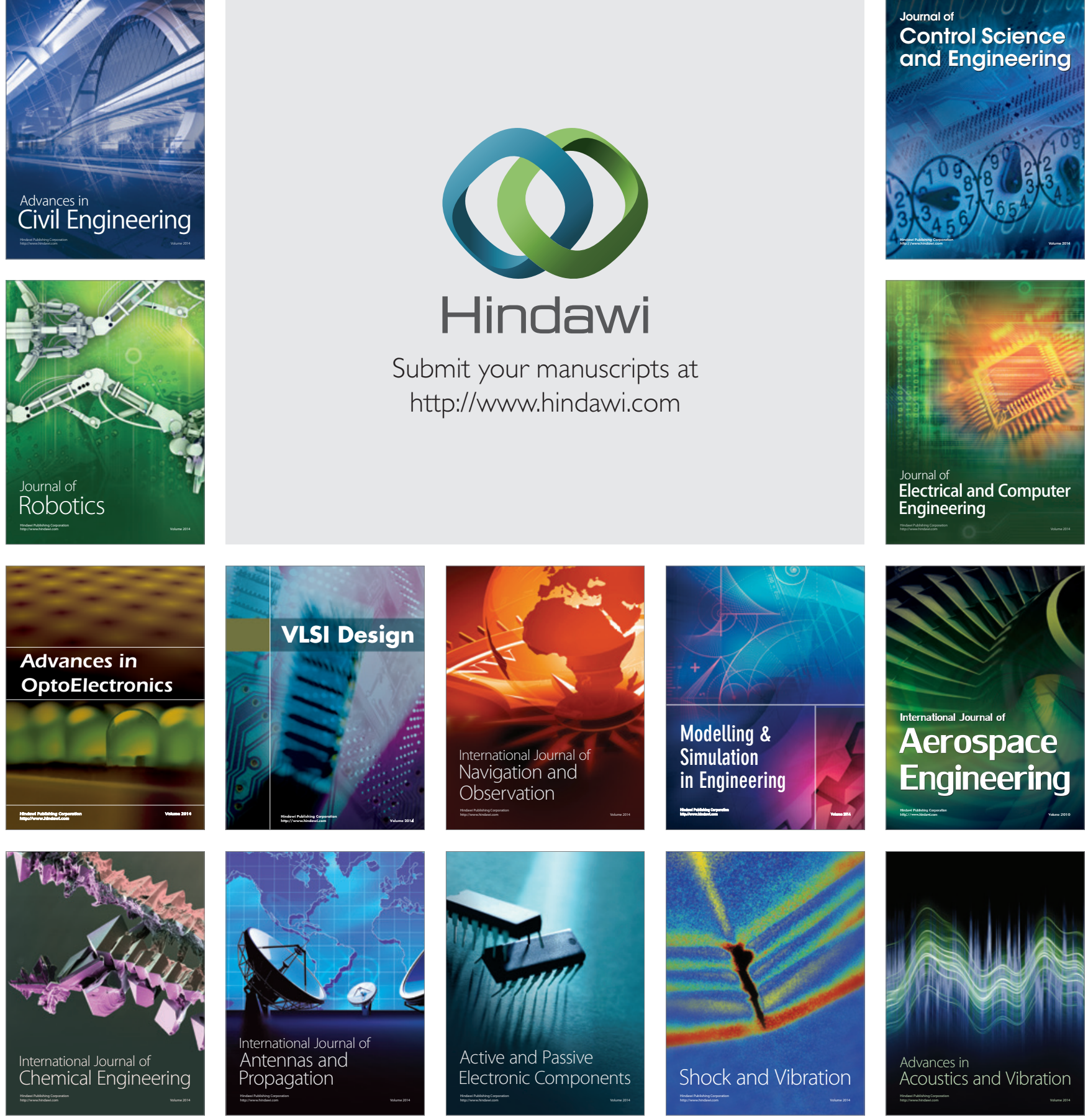